\title{
The use of mandibular advancement devices in obstructive sleep apnea: a proven and effective therapy
}

\author{
Sylvan Mintz ${ }^{1,2}$ \\ Received: 25 June 2018 / Accepted: 8 September 2018 / Published online: 4 October 2018 \\ (C) Springer Nature Switzerland AG 2018
}

Dear Editor,

I am honored that Dr. Sergio Tufik and his team feel my recent research on the use of mandibular appliances in the treatment of obstructive sleep apnea was so well received. As emphasized in my paper and mentioned by Dr. Tufik, the lack of a better system for follow-up sleep studies utilizing oral devices for verifying effectiveness of this therapy is needed. Although his suggestion of a multispecialty team approach with technicians, physicians, speech therapists, physiotherapist, and dentists would be ideal in treating patients with sleep apnea, this is rarely the case, at least in the USA. One of the reasons to publish my results was to demonstrate that in the real world of private practice, the success of customized titratable mandibular appliances for the management of obstructive sleep apnea is extremely high.

My study included all patients regardless of severity of sleep apnea and, with the exception of patients having active temporomandibular disorders and/or completely edentulous, achieved a high degree of success. Not only was mild to moderate sleep apnea successfully managed, but severe patients also achieved good results. My study also demonstrated that a variety of customized, titratable devices were equally effective. I believe better educated and trained dentists in this field are greatly needed. Similar to Dr. Tufik, I am hopeful that more physicians will view oral devices not just as adjunctive therapy, but as potential first-line management for their patients with sleep apnea.

\section{Compliance with ethical standards}

Conflict of interest The author declares that he has no conflict of interest.
Sylvan Mintz

tmjsleep@ verizon.net

1 Department of Pediatrics, George Washington School of Medicine, Washington, DC, USA

2 Bethesda, USA 